\title{
Energy-Efficient Collision Resolution in Wireless Ad-Hoc Networks
}

\author{
Yalin Evren Sagduyu, Anthony Ephremides \\ Electrical and Computer Engineering Dept. \\ and Institute for Systems Research \\ University of Maryland \\ College Park, MD 20742, USA \\ \{sagduyuy, tony\}@eng.umd.edu
}

\begin{abstract}
In this paper, we address the collision resolution (CR) problem from an energy-efficiency point of view and develop a residual-energy-based collision resolution algorithm (CRA) for energy-limited terminals. In this algorithm, which is based on tree-splitting, packets involved in a collision are partitioned into subsets according to the amount of residual battery energy left at the corresponding terminals, and retransmissions are scheduled according to a tree structure. We extend the proposed energybased CR approach to cases without hard energy constraints but, rather, with energy-efficiency objectives. The algorithm then utilizes the distance from the receiver as the criterion. We evaluate the proposed algorithm via simulation for communication systems ranging from simple single-cell classical collision channel models to general multi-hop wireless ad-hoc networks.
\end{abstract}

\section{INTRODUCTION}

In wireless access, whether in cellular networks or general ad-hoc networks, energy-efficiency is of paramount importance. Especially when energy is finite and non-renewable, the performance objectives (throughput, bit-volume, lifetime and delay) are intertwined and involve trade-offs that have not been clearly developed or understood yet. To obtain a good understanding of these trade-offs, we focus first on a simple version of wireless access. That is, we initially strip out all the complexities that multi-hop operation introduces (such as routing and coordination of transmissions over multiple hops for interference avoidance) and concentrate on a single cell with one receiver and multiple transmitters and revisit the well-studied issue of multiple access in this environment from the energy-efficiency perspective. We consider a single communication channel shared by all uplink users and look at the basic form of random access that was proven to have the best stable throughput properties, namely the one based on conflict resolution [1], [2], [3], [4]. We focus on random, rather than scheduled, access because at some level (perhaps at the reservation sub-channel level) some form of random access will be unavoidable in any wireless network.

Prepared through collaborative participation in the Communications and Networks Consortium sponsored by the U.S. Army Research Laboratory under the Collaborative Technology Alliance Program, Cooperative Agreement DAAD19-01-2-0011. The U.S. Government is authorized to reproduce and distribute reprints for Government purposes notwithstanding any copyright notation thereon.

The work presented in this paper was also in part supported by the NSF grant 5-24047 and the ONR grant 5-28623.
The main idea in our paper is to base the criterion for splitting the number of interfering users not on ID [1] or arrival time [2], as was done before, but on the amount of residual energy left at each node. This novel criterion produces surprising performance gains. We consider both the simple classical collision channel model as well as the signal-tointerference-plus-noise-ratio (SINR)-based physical model for packet collision. We then allow mobility (which has significant effect on performance of the SINR-based model) and then, after showing the performance gain achieved, we outline an extension of our approach to multi-hop ad-hoc networks. We consider both the case of finite, non-renewable energy as well as the case of unlimited energy in which, however we require energy-efficient operation.

The paper is organized as follows. Section II is devoted to the energy-limited case for a single cell with a finite number of nodes that have uniformly distributed amounts of initial battery energy. We describe our residual-energy-based treesplitting (REBS) algorithm and compare its performance to the first-come-first-served (FCFS) algorithm as well as to the purely random tree-splitting (RS) algorithm. In section III, we revise the REBS algorithm to make it applicable to the case of nodes with equal amounts of initial battery energy. Section IV introduces a new energy-efficiency criterion for conflict resolution in systems without hard energy constraints and describes a distance-based tree-splitting (DBS) algorithm. This is followed by the extension of our approach to multihop ad-hoc networks in section V. In section VI, we study the steady-state stability issues in CRA's from perspective of throughput and delay. Finally, we draw some conclusions and outline thoughts for future work in section VII.

\section{Collision Resolution in Energy-Limited AcCess WITH A FINITE NUMBER OF USERS - UNIFORM DISTRIBUTION OF INITIAL ENERGY}

\section{A. Motivation for Energy-Limited Collision Resolution}

In the well-known tree-splitting CRA's [2], all packets involved in a collision are partitioned into subsets that attempt transmissions simultaneously in subsequent time slots according to identity or time-of-arrival criteria. Thus it is possible that energy-poor nodes waste additional energy in subsequent unsuccessful attempts. We propose a modification 
of this partitioning philosophy so that packets are split on a CR tree according to the amount of residual battery energy left at the corresponding transmitters. Thus the simultaneous contention of low- and high-energy nodes is restricted and, hence, a reduction is expected in the probability that lowenergy nodes waste their limited energy for unsuccessful retransmission attempts. In the sequel, we expect familiarity of the reader with the tree structure of CRA's as explained in [2] and [4]. The reasons that our approach is expected to yield longer node lifetimes and improved performance are as follows.

If the left subset that has been formed at the first splitting after a collision results in another collision, the corresponding right subset is combined with waiting new arrivals, which leads to an improvement in throughput. If this particular method, known as tree pruning [2], [4], is employed in any CRA, some packets that lie on right branches in the $\mathrm{CR}$ tree structure will be probably incorporated into the next collision resolution period (CRP) and consequently the particular nodes transmitting these packets will participate in fewer packet transmissions. If packets are generated according to a Poisson distribution, they join left and right branches with equal probability in the FCFS and RS algorithms and consequently all nodes on the average participate in the same number of transmissions independent of the amount of their residual energy. However, in our energylimited CR approach, the probability that a packet in the left subset belongs to a high-energy node is strictly larger compared to the opposite case, i.e. that it originated from a node with a low amount of energy. Therefore, high-energy nodes will be typically involved in more packet transmissions during any CRP, while low-energy nodes will be spared some of the unsuccessful attempts. Thus an increase on the average lifetime is expected.

In addition, when an idle slot follows a collision during a CRP, the right subset to be transmitted next is immediately split into two sets prior to transmission and only the newly formed left subset is allowed to transmit. Since the probability that this subset contains at least two packets is equal to one, this modification, which is known as level skipping [2], [4], increases throughput by preventing collisions that would occur otherwise. Since packets of energy-poor nodes tend to be on right subgroups at each tree splitting according to our energy-limited CR approach, the level skipping method mainly protects the low-energy nodes against packet collisions. A similar energy-efficient result cannot be achieved in other CRA's, since nodes are distributed on branches according to criteria (e.g. ID, time of arrival) that are independent of residual energy level.

\section{B. Model Description}

We consider a single-cell system with one receiver and multiple uplink transmitters sharing a single, slotted, synchronous collision channel. All transmitted packets have the same length and require one time unit (equal to one time slot) for transmission. Packets arrive at nodes according to independent Poisson processes with aggregate arrival rate of $\lambda$ (packets/slot). Nodes are equipped with omni-directional antennas and use fixed amount of power for every attempted packet transmission. In this section, we assume that initial residual energy levels of nodes are independently and uniformly distributed between zero and a common value $E_{\max }$ of maximum battery energy. This assumption will be relaxed in subsequent sections.

The transmitter nodes involved in a CRP have immediate access (for the moment we neglect propagation or other delays) to error-free ternary feedback, i.e. whether a collision (e), a success (1) or an idle (0) was observed during the preceding slot. This allows the nodes to run individual copies of the CRA in a distributed fashion. Packets arriving at each node are stored in a queue operating on first-come-first-served basis without any buffer size constraint. Throughout this paper, two separate models are considered for the channel, namely, (i) the classical collision channel model, and (ii) the physical model.

In the classical collision channel, idle, success and collision refer to the cases when 0,1 or more than one packet are transmitted during a particular time slot. The simplified successful packet reception criterion dictated by the classical collision channel can be replaced by a more realistic SINRbased physical model [5]. According to the physical model, the transmission of node $i$ is successfully received by node $j$, if

$$
\frac{\frac{P_{i}}{\max ^{\alpha}\left(r_{0}, r_{i, j}\right)}}{N+\sum_{k \neq i, k \in \tau} \frac{P_{k}}{\max ^{\alpha}\left(r_{0}, r_{k, j}\right)}} \geq \beta
$$

where $r_{i, j}$ is the distance between nodes $i$ and $j, P_{i}$ is the transmission power of node $i, N$ is the ambient noise power and $\tau$ denotes the subset of nodes simultaneously transmitting in a given time slot. Signal power decays with distance $r$ as $\frac{1}{r^{\alpha}}$ in the far-field region (beyond a distance of $r_{0}$ from the transmitter) with path loss exponent $\alpha \geq 2$. This models a situation where the minimum SINR of $\beta$ is necessary for successful packet reception. In the physical case model, it is possible to have success and collision in the same slot. So we assume that each node participating in the contention is informed of the ID of the successful packet(s).

\section{Residual-Energy-Based Tree-Splitting (REBS) Collision Resolution Algorithm}

During the CR process, at each integer time $k$, the residualenergy-based tree-splitting (REBS) algorithm we propose in this paper specifies the packets to be transmitted in slot $k$ to be the set of all packets that arrived at nodes with residual energy values between $T(k)-\alpha(k)$ and $T(k)$. During a CRP, each node attempts to successfully deliver at most one packet from its current temporal allocation window and subsequently all nodes with empty queues shift their temporal windows by a fixed amount at the end of the CRP to accept new packets. In the REBS algorithm, nodes with higher amount of residual energy transmit first (i.e. their packets are assigned to the left branch of the CR tree), whereas other nodes wait until the high-energy nodes successfully retransmit. In this way, we eliminate the simultaneous contention of low-energy nodes with high-energy ones for the same channel.

We let $\sigma(k)$ indicate whether nodes transmitting in slot $k$ are on the left or the right branch of the tree. The REBS 
algorithm specifies the parameters $T(k), \alpha(k)$ and the status $\sigma(k)=R$ or $L$ in terms of the feedback on slot $k$, and on the previous values of the parameters $T(k-1)$, $\alpha(k-1)$ and $\sigma(k-1)$. Initially (at the $0^{t h}$ slot) just before the first transmission initiating the CR process, we have $T(0)=E_{\max }, \alpha(0)=E_{\max }, \sigma(0)=R$. During a CRP, the algorithm parameters evolve as follows:

If feedback $=\mathrm{e}$, we have

$$
T(k)=T(k-1)-1, \alpha(k)=\left\lfloor\frac{\alpha(k-1)}{2}\right\rfloor, \sigma(k)=L
$$

If feedback $=1$ and $\sigma(k-1)=L$, we have

$$
\begin{gathered}
T(k)=T(k-1)-\alpha(k-1), \\
\alpha(k)=\alpha(k-1)-1, \sigma(k)=R
\end{gathered}
$$

If feedback $=0$ and $\sigma(k-1)=L$, we have

$T(k)=T(k-1)-\alpha(k-1), \alpha(k)=\left\lfloor\frac{\alpha(k-1)}{2}\right\rfloor, \sigma(k)=L$

If feedback $=0$ or 1 and $\sigma(k-1)=R$, we have

$$
\begin{gathered}
T(k)=T(k-1)-\alpha(k-1), \\
\alpha(k)=T(k), \sigma(k)=R
\end{gathered}
$$

The usual Markov analysis for stability and throughput of a CRA [2] is not applicable here, since there is no steady state but, rather, a transient phase until all nodes run out of energy supply.

\section{Description of the Simulation Environment}

In this section, we evaluate the performance of the simple form of the REBS, FCFS and RS algorithms for both the classical collision channel as well as the physical model. To resolve packet collisions, the FCFS algorithm bases the criterion for splitting on time of packet arrival, whereas the RS algorithm schedules transmissions by splitting packets randomly (e.g. by flipping a fair coin) on a CR tree. The wireless single-cell system simulated in this section consists of one receiver and $m=10$ transmitters. Packets are assumed to arrive at each node according to a Poisson process with arrival rate of $\lambda / m . \lambda$ is chosen to be 0.5 (packets/slot). Nodes are initially uniformly distributed on a circular area of $10^{4}$ (unit length square). The packet transmission power is fixed and the initial energy levels of all nodes are uniformly distributed between 0 and $E_{\max }=100$ (we assume that each packet transmission utilizes one unit of energy). The temporal allocation interval $\alpha_{0, T}$ is 2 time slots and the initial energy allocation interval $\alpha_{0, E}$ is equal to $E_{\max }$. In the physical model, the SINR threshold $\beta$ is $6 \mathrm{~dB}$, path loss exponent $\alpha$ is 2 , near-field region radius $r_{0}$ is one unit length and thermal noise is additive white Gaussian with variance $10^{-5}$.

Although the classical collision channel output is independent of the mobility patterns observed in the network, the successful packet reception in the physical model depends on the distance between transmitter and receiver. We specify the motion of nodes in a bounded planar region by a continuoustime random process based on mobility epochs, as proposed in [6]. The $i^{\text {th }}$ mobility epoch is defined to be a random
TABLE I

SySTEM PARAMETERS FOR SiMULATIONS

\begin{tabular}{|c|c|}
\hline Number of Transmitters $m$ & 10 \\
\hline Circular Network Area & $10^{4}$ \\
\hline Exp.Val.of Node Velocity $\mu_{n}$ & $0.5 /$ slot \\
\hline Exp.Val.of Mobil.Epoch Length $\lambda_{n}^{-1}$ & 5 slots \\
\hline Packet Interarrival Time $\lambda^{-1}$ & 2 slots \\
\hline Maximum Battery Energy $E_{\max }$ & 100 \\
\hline SINR Threshold $\beta$ & $6 \mathrm{~dB}$ \\
\hline Path Loss Exponent $\alpha$ & 2 \\
\hline Antenna Near-Field Region Radius $r_{0}$ & 1 \\
\hline Transmission Power P & $10^{-5}$ \\
\hline Noise Variance $N$ & 2 slots \\
\hline Temporal Allocation Interval $\alpha_{0, T}$ \\
\hline
\end{tabular}

length interval $T_{i, n}$ during which a node $n$ moves in a constant direction $\theta_{i, n}$ at a constant speed $v_{i, n}$. To model the mobility, the following assumptions are made: (A1) Mobility epoch lengths are exponentially distributed with mean $\lambda_{n}^{-1}$, i.e. change in mobility patterns is characterized as Poisson process, (A2) $\theta_{i, n}$ is independent and uniformly distributed in $[0,2 \pi],(\mathrm{A} 3) v_{i, n}$ is independent and identically distributed with mean $\mu_{i, n}=\mu_{n}$ and variance $\sigma_{i, n}^{2}=\sigma_{n}^{2}$, for all $i$, and (A4) $\theta_{i, n}, v_{i, n}$ are uncorrelated for all $i, n$. In our simulations, $v_{i, n}$ is uniformly distributed between 0 and 1 (unit length/time slot). The expected mobility epoch length $\lambda_{n}^{-1}$ is 5 (time slots). The system parameters and their values in our simulations are summarized in Table I.

\section{E. Performance Results}

The following measures are used to evaluate the performance of the FCFS, RS and REBS algorithms:

- System Lifetime is defined as the length of the time interval from the beginning of the simulation until the first time instant when the number of nodes with transmission energy of more than or equal to one unit drops below two, i.e. there is no live transmitter-receiver pair left in the network.

- First Death Time denotes the length of the period from the beginning of the simulation until the first time when the battery energy of a node drops below one unit energy.

- Throughput is defined as the total number of successfully transmitted packets per unit time (considered only until the first node death time).

- Energy Cost denotes the battery energy spent per successfully delivered packet over system lifetime.

- CRP Length is the average length of time interval required to resolve a collision.

- Volume $(t)$ is defined as the number of successfully transmitted packets over time interval $[0, t]$.

- Average Packet Delay, D, denotes the delay that a packet experiences from the time instant it enters the queue of the transmitting node until it is successfully received by the intended receiver.

System lifetime, first node death time, CRP length and average packet delay are measured in time slots. To adequately suppress the effect of unlikely fluctuations of minor 
importance in the network on performance evaluation, all results have been averaged over 100 different topologies, initial energy distributions, mobility and packet traffic patterns. Table II shows simulation results for the classical collision channel model. The delivered packet volume and the average packet delay as a function of the total packet arrival rate of the system are shown in Figure 1 and Figure 2, respectively. Simulation results verify the superior performance of the REBS algorithm in the case of uniform distribution of individual initial energy levels. If capture of packets is possible (as in the physical model), the probability of successful reception increases and all algorithms perform better than in the classical collision channel, as shown in Table III and Figure 3.

TABLE II

Classical Collision Channel Model

\begin{tabular}{|c||c|c|c|}
\hline & FCFS & RS & REBS \\
\hline Throughput & 0.4474 & 0.4393 & 0.4535 \\
\hline CRP Length & 1.5803 & 1.6002 & 1.4002 \\
\hline Energy Cost & 1.5802 & 1.6106 & 1.4393 \\
\hline System Lifetime & 3838 & 3481 & 4272 \\
\hline First Death Time & 330 & 308 & 378 \\
\hline
\end{tabular}

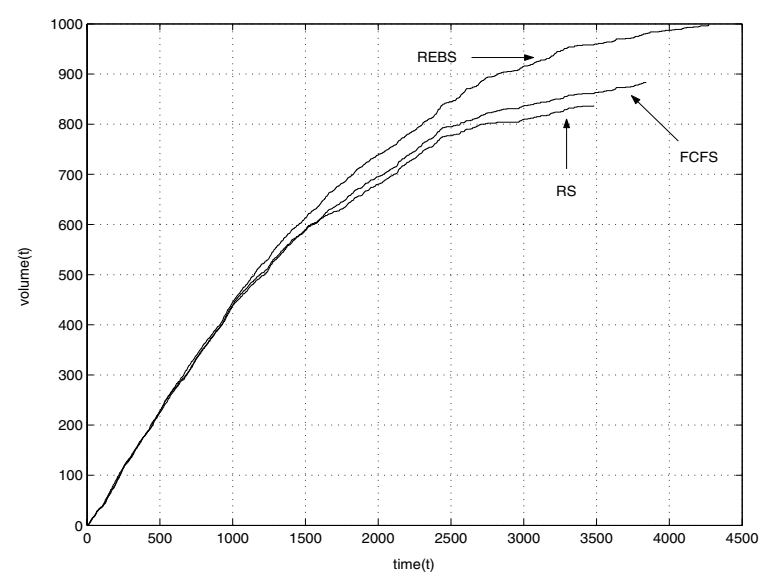

Fig. 1. Evolution of cumulative delivered packet volume in the classical collision channel model

TABLE III

PHYSICAL MODEL

\begin{tabular}{|c||c|c|c|}
\hline & FCFS & RS & REBS \\
\hline Throughput & 0.4558 & 0.4434 & 0.4773 \\
\hline CRP Length & 1.4911 & 1.5002 & 1.4045 \\
\hline Energy Cost & 1.3987 & 1.5400 & 1.3016 \\
\hline System Lifetime & 3976 & 3731 & 4447 \\
\hline First Death Time & 384 & 323 & 433 \\
\hline
\end{tabular}

\section{Collision Resolution in Energy-Limited ACCESS With Finite Number of USERS - EQUAL INITIAL ENERG C CASE}

In the case of equal initial energy, the performance of the proposed version of the REBS algorithm is expected to be poor, as shown in Table IV and Figure 4, since it is designed

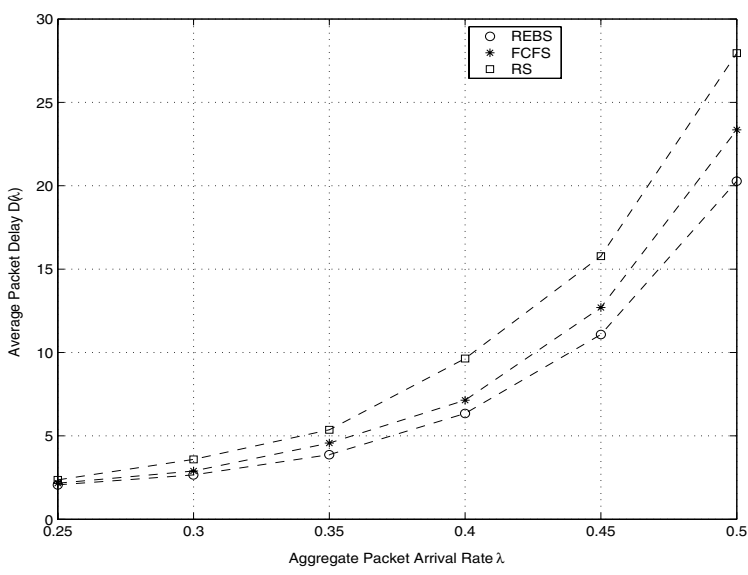

Fig. 2. Average packet delay as a function of the aggregate packet arrival rate in the classical collision channel model

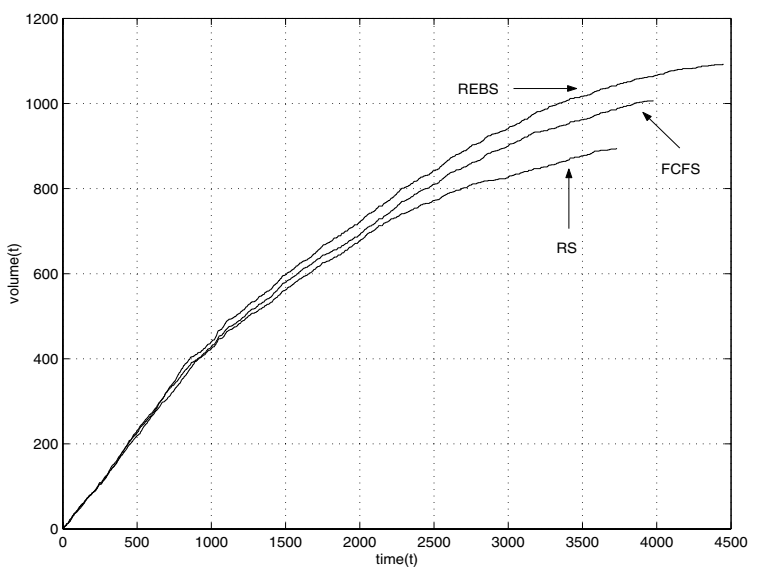

Fig. 3. Evolution of cumulative delivered packet volume in the physical model

to exploit a spread of the residual energy values among the nodes. To deal with the performance degradation in the case of equal initial battery energy, we propose to employ initially the FCFS algorithm and postpone the use of the REBS algorithm until the residual energy levels of nodes are sufficiently spread. There remains the open question of how to optimally merge the FCFS and REBS algorithms with this hybrid CR method.

TABLE IV

Equal Initial Energy Case \& Classical Collision Channel MODEL

\begin{tabular}{|c||c|c|c|c|}
\hline & FCFS & RS & REBS & HYBRID \\
\hline Throughput & 0.4613 & 0.4558 & 0.4061 & 0.4696 \\
\hline CRP Length & 2.1230 & 2.5326 & 2.3545 & 2.0925 \\
\hline Energy Cost & 2.0097 & 2.0556 & 2.2441 & 1.9792 \\
\hline System Lifetime & 1688 & 1527 & 1460 & 1939 \\
\hline First Death Time & 1079 & 997 & 1039 & 1146 \\
\hline
\end{tabular}

If we assume that nodes generate packets with different rates, residual energy levels of nodes are faster spread away and quickly approach uniform distribution. The effect of nonuniform packet traffic is illustrated in Figure 5, where the 
packet generating rate of node $i$ is $\lambda_{i}=\lambda_{\frac{2 i}{m(m+1)}}$, for $1 \leq i \leq m$. Also, if we allow the system to drop packets that cannot be resolved in a sequence of CRP's in order to avoid the proliferation of packets in queues, the performance of the REBS algorithm becomes better and even comparable to that of the other algorithms in the long run, as illustrated in Figure 6. In this section, simulations are performed only for the classical collision channel, $\lambda=0.5$ and $E_{\max }=50$.

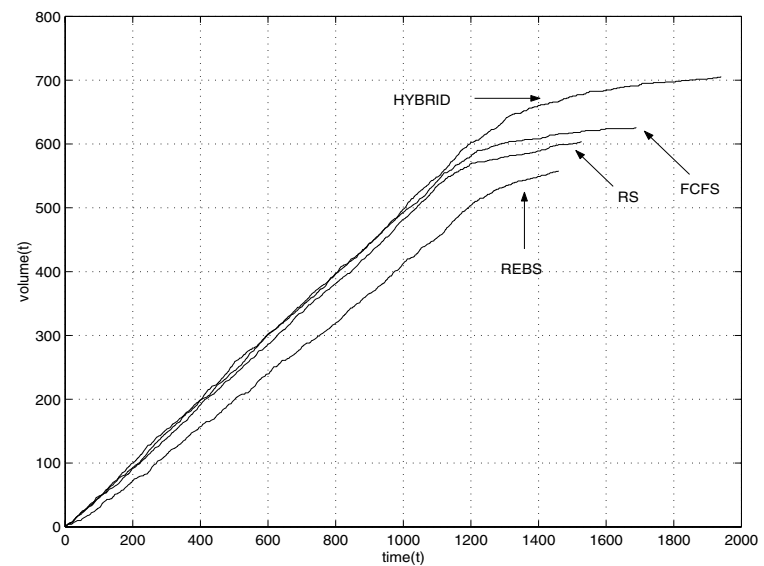

Fig. 4. Evolution of cumulative delivered packet for the equal initial energy case in the classical collision channel model

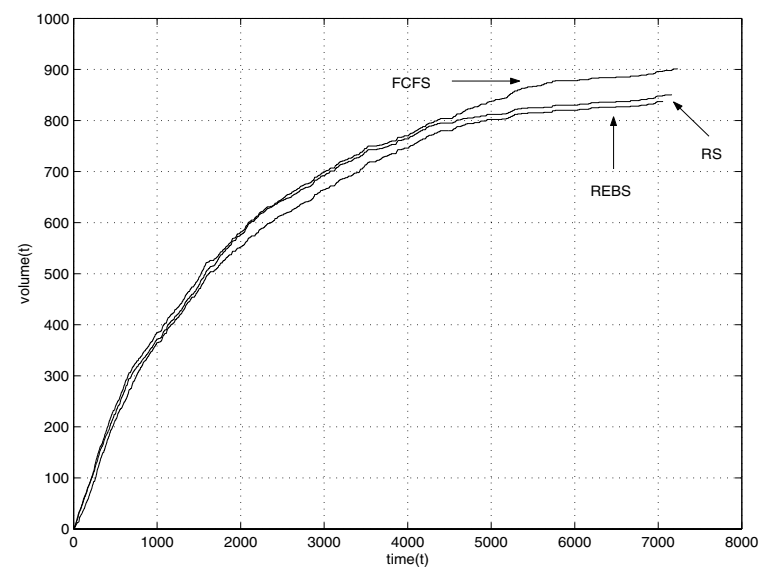

Fig. 5. Evolution of cumulative delivered packet volume for the equal initial energy case with non-uniform packet traffic in the classical collision channel model

We continue, however, our comparisons with our original assumption of uniformly generated traffic without packet dropping. A simple method to implement a hybrid CRA would be to use first the FCFS algorithm and abruptly switch to the REBS algorithm after a predetermined number of time slots or whenever the residual energy in the system drops below a particular threshold.

A more dynamic solution that we prefer is to gradually interchange between the FCFS and REBS algorithms by employing a quaternary splitting and using time and energy windows simultaneously. Accordingly, if a collision occurs, first high-energy nodes transmit their packets according to their time of arrival. This is followed by transmissions of

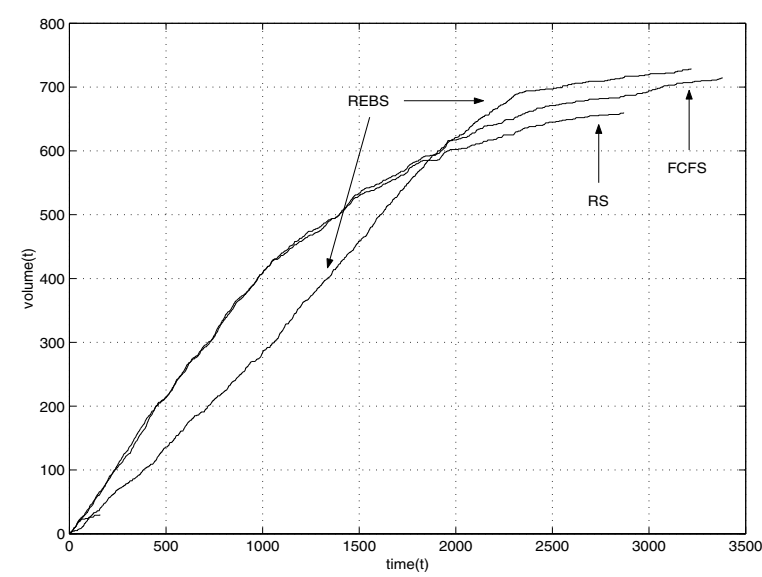

Fig. 6. Evolution of cumulative delivered packet volume for the equal initial energy case with packet dropping in the classical collision channel model

old and new packets by low-energy nodes in subsequent time slots, while high-energy nodes are waiting. We use the same evolution rules for time and energy window parameters, as described in the FCFS and REBS algorithms, but we split packets involved in a collision into four branches instead of two as we did before.

The hybrid CRA starts with a very small $\alpha_{0}$ for energy window, i.e. we initially use an algorithm similar to the FCFS algorithm to cope with the equal battery energy assumption. As the algorithm progresses, we increase $\alpha_{0}$ in energy window to $E_{\text {max }}$, while gradually decreasing the number, which divides the allocation interval length $\alpha$ in temporal window at each splitting, from 2 to 1 , i.e. we gradually allow nodes to join only the left subset in the CR tree corresponding to the temporal window and ignore the associated right subsets. Eventually, we end up with a CRA not distinguishable from the REBS algorithm. The gradual changes we impose on the window parameters can be implemented as a function of time or system residual energy. This hybrid method is compared to the FCFS, RS and REBS algorithms and the performance gains obtained at the expense of increased computational complexity are illustrated in Figure 4 and Table IV.

\section{ENERgy-EFFicient COLLISION RESOLUTION - UNLIMITED (OR RENEWABLE) ENERGY CASE}

In this section, we consider the case of either a hypothetical network without energy constraints, or equivalently, a more realistic network with immediately renewable energy resources. In this context, a CRA is called energy-efficient, if it helps to reduce the energy consumption of the individual nodes per successfully transmitted packet. For our purposes, it is sufficient to assume that each node can estimate the distance to its intended receiver (rather than the exact geographical node position). Sharing location information among nodes with correlated mobility patterns or using global positioning system can be cited as some of the possible solutions to the distance estimation problem. However, the precise method of distance estimation is outside the scope of this paper.

According to the signal propagation law embedded in the physical model, the received signal power of a transmitting 
node decays with distance $r$ as $\frac{1}{r^{\alpha}}$ with $\alpha \geq 2$ in the farfield region of the transmitter. In this section, we assume the SINR-based physical model as the only successful packet reception criterion. In the case of fixed transmission power, the probability of packet error will decrease, if nodes closer to their intended receivers are given higher transmission priority. Similarly, in the case of variable transmission power, the total energy consumption required to achieve a particular packet error rate will decrease, if we order the packet transmissions according to the distances between the corresponding transmitter and receiver nodes. The idea behind this criterion is similar to the one used in our original simple version of the REBS algorithm; namely, the nodes that are enabled to transmit first are, on average, more likely to be involved in energy-wasting collisions. Thus, by enabling the nodes that are close to the receiver first, we end up wasting less energy. The nodes that are far (and, hence, need higher power to reach the receiver) are shielded somewhat from the first group of (more) frequent collisions.

This approach is consistent with an important result from the area of multiuser diversity, which states that the information capacity is achieved by letting only the user with the best channel transmit at any given time [7]. According to the physical model, users with best channels are the nodes that are closer to their respective receivers and they are given higher priority in the transmission order in our tree-splitting-based CR approach. The relative distances and consequently the channel conditions undergo changes depending on the mobility patterns observed in the network. For better performance results, the node mobility should be fast enough to create a dynamic priority list for the transmission order. Otherwise, packets of nodes that are initially far away from their intended receivers will face undesirably long delays. On the other hand, nodes should move slowly enough relative to the conflict resolution rate of the system to avoid distortions in the general structure of the CR tree.

During the CR process, at each integer time $k$, the distancebased tree-splitting (DBS) algorithm specifies the packets to be transmitted in slot $k$ to be the set of all packets that arrived at nodes with distance to their intended receivers between $r(k)$ and $r(k)+\alpha(k)$ (or with required energy consumption to achieve a desired packet error rate between $E(k)$ and $E(k)+$ $\beta(k)$, where $E(k)=K r^{2}(k)$ and $\beta(k)=2 \sqrt{K E(k)} \alpha(k)+$ $K \alpha^{2}(k)$, for some fixed constant $\left.K\right)$. Evolution of $r(k)$ and $\alpha(k)$ during the DBS algorithm is similar to the evolution of window parameters in the REBS and FCFS algorithms.

For the unlimited energy case, throughput needs to be redefined as the number of successfully delivered packets per unit time measured over the entire simulation (since no nodes die in this case). We assume that nodes can autonomously adjust their transmission power to the minimum value that would be required to exceed the SINR requirement if no interference was present. Clearly, if the value is set at a higher level, some interference may be tolerated at the expense of higher energy consumption. We did not investigate here this trade-off but we point at a detailed study in [8]. Simulations are conducted over 5000 time slots and Table V shows the results of the performance evaluation of the DBS, FCFS and RS algorithms under the assumptions of the physical model, unlimited (or immediately renewable) battery energy and variable transmission power.

TABLE V

Unlimited Energy \& Physical Model

\begin{tabular}{|c||c|c|c|}
\hline & FCFS & RS & DBS \\
\hline \hline Throughput & 0.4758 & 0.4634 & 0.4863 \\
\hline CRP Length & 1.4911 & 1.5002 & 1.4045 \\
\hline Energy Cost & 1.3987 & 1.5400 & 1.3016 \\
\hline
\end{tabular}

\section{Collision Resolution in Multi-Hop AD-HoC NETWORKS}

\section{A. Joint Operation of Collision Resolution Trees}

Wireless ad-hoc networks typically consist of multiple transmitter-receiver pairs and there would be several simultaneous CR processes taking place at different receiver nodes, if we were to use conflict resolution methods for resolving contention. In this section, we analyze the interdependence between $C R$ processes in a simple network with two receiver nodes as depicted in Figure 7 . In this context, we outline a method to reduce the coupling between two interacting CR processes by using the fact that the nodes in the reception range of both receiver nodes have access to the feedback information about the status of both $\mathrm{CR}$ processes. We realize that this limited goal falls short of the objective of fully extending our approach to general ad-hoc networks. However, we believe that we need to understand the CRA interactions better before we can extend them.

We assume that the transmission range of any node is circular with radius $r$ and beyond that range no interference is caused as opposed to the physical model. The aggregate effect of individually negligible amount of interference caused by very large number of far interferers might distort an ongoing transmission. However, we restrict the analysis in this section to small networks and continue with the bounded transmission range assumption for the purpose of simplicity. According to the Figure 7, there are four different types of transmitting nodes. Nodes of type A1 and A2 are in the reception range of only the receiver nodes $\mathrm{R} 1$ and $\mathrm{R} 2$, and intend to transmit to $\mathrm{R} 1$ and R2, respectively. On the other hand, nodes of type B1 and $\mathrm{B} 2$ lie in the intersection of the reception ranges of both receiver nodes and their packets are destined to the receiver nodes R1 and R2, respectively.

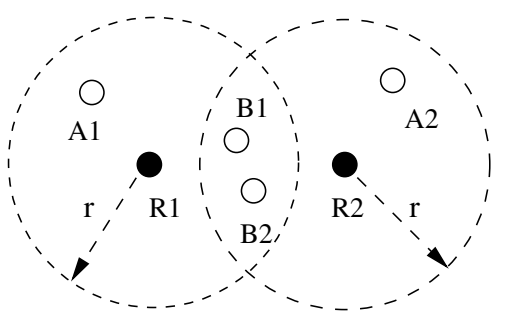

Fig. 7. The simple network model used to analyze the joint operation of simultaneous collision resolution processes 
The only possible effect of nodes of type B1 and B2 on the $\mathrm{CR}$ process observed at the receiver nodes $\mathrm{R} 2$ and $\mathrm{R} 1$ is a possible change of a successful reception to a collision. Therefore, each CR process has the negative impact of slowing down the other simultaneous $\mathrm{CR}$ processes, provided that the reception ranges of the corresponding receivers overlap. CRA's discussed so far can be still used individually at each receiver node without a need for synchronization between different CR processes, if additional delays and performance degradation with respect to throughput and energy-efficiency criteria are tolerable.

Let $k$ be the last parameter update time of our simple version of the partial CR tree decoupling algorithm. Without loss of generality, we assume that $T_{1}(k) \geq T_{2}(k)$ at the $k^{t h}$ iteration of the CRA. Then, nodes of type A1 and A2 start operating with the CRA parameter pairs of $\left(T_{1}(k+1), \alpha_{1}(k+1)\right)$ and $\left(T 2(k+1), \alpha_{2}(k+1)\right)$, respectively. Nodes of type B1 and B2 first use the dual feedback that they receive from $\mathrm{R} 1$ and $\mathrm{R} 2$ to determine $T 1(k+1), T 2(k+1), \alpha_{1}(k+1)$ and $\alpha_{2}(k+1)$. Then, they individually perform a nonlinear mapping from $\left(T 1(k), T 2(k), \alpha_{1}(k), \alpha_{2}(k)\right)$ to $\left(T 3(k+1), \alpha_{3}(k+1)\right)$. As a result, nodes of type B1 participate in the CR process assuming the parameter pair $\left(T_{3}(k+1)=T_{1}(k), \alpha_{3}(k+1)\right)=$ $\left.T_{3}(k+1)-\max \left(T_{2}(k), T_{1}(k)-\alpha_{1}(k)\right)\right)$, while nodes of type $\mathrm{B} 2$ remain idle.

The CR tree corresponding to the receiver node $\mathrm{R} 1$ is completely decoupled from the other tree, if we employ the proposed decoupling method. However, the CR process at R2 still depends on the other CR process through the transmissions of nodes of type B1. Therefore, the average number of nodes interfering at $\mathrm{R} 1$ is smaller than the average number of nodes interfering at R2, provided that transmitters are uniformly distributed on the union of the reception ranges of both receiver nodes. Hence, the $\mathrm{CR}$ process at $\mathrm{R} 2$ is slower than the other $\mathrm{CR}$ process and it is very likely that $T_{1}$ eventually drops below $T_{2}$, which necessitates an update of type 1 .

Update 1: If $T_{2}(j)>T_{1}(j)+c_{1}$, for some constant $c_{1}$ and time instant $j$, we have

- Nodes of type A2 start transmitting and nodes of type A1 remain idle.

- $T_{1}(j+1)=T_{3}(j)$ and $\alpha_{1}(j+1)=\alpha_{3}(j)$

Nodes of type B2 may face extensive delays, if $T_{1}$ cannot reach $T_{2}$ within a reasonable time interval. Therefore, it might be necessary to couple both trees to slow down the CR process at the receiver node $\mathrm{R} 2$, which can be achieved by an update of type 2 .

Update 2: If $T_{2}(j)<c_{2}<T_{1}(j)$, for some constant $c_{2}$ and time instant $j$, we have

- $T_{2}(j+1)=T_{3}(j)$ and $\alpha_{2}(j+1)=\alpha_{3}(j)$

If the CR process for the packets of nodes of type B1 is faster than that of nodes of type A1, $T_{1}$ may drop below $T_{3}(k)-\alpha_{3}(k)$ and the roles of waiting and transmitting nodes need to be exchanged by an update of type 3 .

Update 3: If $T_{1}(j)<T_{3}(k)-\alpha_{3}(k)+c_{3}$, for some constant $c_{3}$ and last update time $k$, we have
- Nodes of type A2 start transmitting and nodes of type A1 remain idle.

- $T_{1}(j+1)=T_{3}(j)$ and $\alpha_{1}(j+1)=\alpha_{3}(j)$

The algorithm should stop, when $\max \left(T_{1}, T_{2}\right)=0$. Performing periodically the nonlinear mapping operation from $\left(T_{1}(k), T_{2}(k), \alpha_{1}(k), \alpha_{2}(k)\right)$ to $\left(T_{3}(k), \alpha_{3}(k)\right)$ will increase the reliability of the CRA parameter information. This algorithm promises performance gains at the expense of increased complexity and is desirable in that it preserves the distributed nature of previous algorithms and relies only on the conventional feedback information.

\section{B. Description of the Simulation Environment - Multi-hop Ad- Hoc Network}

As the system model, we assume a mobile wireless adhoc network of $m=10$ nodes with uniformly distributed initial energy levels. Since there is only a single transceiver available at each node, self-interference prohibits simultaneous transmission and reception of nodes. We assume that nodes with less than one unit battery energy cannot transmit or receive packets. Packets are generated by nodes according to independent Poisson distributions with total arrival rate of $\lambda=0.5$ and destined equally likely to any of the remaining $m-1$ nodes. Throughput is defined as the number of packets successfully delivered to destination nodes per unit time without giving any credit to relay traffic.

Direct transmission assumed so far is known to be inefficient from both perspectives of throughput and energy and in some cases even not possible due to the battery power constraints in wireless ad-hoc networks. Therefore, we focus on multi-hop communication among nodes, which employ the distributed Bellman-Ford algorithm for routing of their packets. The transmission range of each node is circular with radius $r=5$. Periodic updates of routing tables are needed for better performance, since the network has a non-stationary nature due to the mobility and the limited energy resources of nodes. To be consistent with our primary objective to preserve transmission energy and extend battery lifetime, the cost of a link (i.e. the cost of a packet transmission) from node $i$ to node $j$ is chosen to be

$$
d_{i j}=\left\{\begin{array}{cl}
\left(\frac{E_{\max }}{E_{j}}\right)^{\gamma} \max ^{\alpha}\left(r_{0}, r_{i, j}\right) & , \text { if } E_{i}>0 \\
\infty & , \text { otherwise }
\end{array}\right.
$$

where $E_{\max }$ is the maximum battery energy, $E_{i}$ is the residual battery energy of node $i, r_{i, j}$ is the distance between nodes $i$ and $j$ and $\gamma$ is a parameter useful for reflecting the importance of residual energy with respect to other term in (6), namely $\max ^{\alpha}\left(r_{0}, r_{i, j}\right)$, which characterizes the energy-efficiency in routing decisions. The specific link metric $d_{i j}$ discourages the inclusion of energy-poor nodes as well as directional links with low probability of successful packet transmission (i.e. between relay nodes far away from each other) in routing decisions.

Since CR processes at different receivers are continuously interacting with each other, the standard structure of the CR tree associated with each receiver node is distorted due to the extra interference caused by packets, which are intended 
for other receiver nodes. The interdependence between CR processes may lead to instability in infinite energy systems. Although networks with finite lifetimes do not suffer from instability problems, they cannot achieve performance gains as desirable as in single-cell systems, because the feedback information is no more reliable due to interference from other CR processes. To solve this problem partially, we apply the specific tree decoupling method we have proposed in section V-A. The simulation results for multi-hop networks are demonstrated in Table VI and Figure 8.

TABLE VI

Multi-HOP AD-Hoc NeTwork Model

\begin{tabular}{|c||c|c|c|}
\hline & FCFS & RS & REBS \\
\hline Throughput & 0.1964 & 0.1782 & 0.1847 \\
\hline CRP Length & 1.8936 & 2.0917 & 1.8847 \\
\hline Energy Cost & 2.1461 & 2.2490 & 2.1377 \\
\hline System Lifetime & 3246 & 3117 & 3832 \\
\hline First Death Time & 303 & 283 & 315 \\
\hline
\end{tabular}

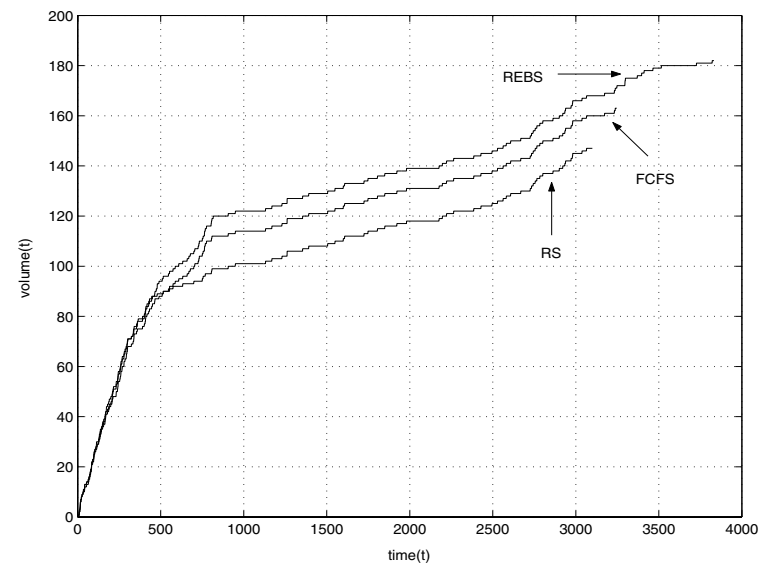

Fig. 8. Evolution of cumulative delivered packet volume in the multi-hop ad-hoc network model

Of course what remains to be done is to compare the proposed CRA-based access control to other methods before concluding that CRA's are the preferred form of access. Such a comparison would require extensive simulations and is outside the scope of this paper. Rather, our objective here is to show that our energy-based ideas as applied to CRA forms of access can be feasibly applied to full-fledged multi-hop adhoc networks.

\section{Queueing Theoretic Stability Analysis of Collision Resolution Processes}

In this section, we revisit the basic single-cell paradigm of CRA-based access for the purpose of proposing delay and stability analysis when the packets that are generated at each node are queued. There is a large volume of related work [9], [10], [11], [12] but none fits exactly our model. Such an analysis is necessary and important because we need to specify how the arriving packets are treated at each node. Generic CRA analyses do not address this question and thus their stability analysis cannot be performed without resolving this issue.

We consider packet collision resolution in systems with a finite number of nodes as opposed to the conventional model of infinite node population, which is extensively studied before in [2], [13] and [14], where each newly arriving packet arrives at a "new" node. We follow a queueing theoretic approach (similar to the one suggested in [15]) and model each node as a queue. The server in this model may be regarded as the intended receiver and the corresponding service for a user is the collision resolution of its packets. The stationary analysis that is needed to study stability and delay cannot be applied to systems with limited energy. However, it is important to understand the behavior of CRA's in systems with a finite number of nodes. It is hoped that the steady-state analysis of these systems provides insights into the transient behavior in the case of finite node energy.

Let $m$ be the total number of nodes in the system and $\lambda$ be the rate of the aggregate Poisson arrival traffic, as defined before. Let $\mu$ denote the service rate (inverse of the expected length of one CRP) and $p^{*}$ the probability that a packet cannot be successfully transmitted during a CRP (hence, the probability that the packet returns back to the queue to be retransmitted in the next CRP). If a CRA employs the tree pruning method as described in section II-A, the corresponding quantity of $p^{*}$ is nonzero.

Every CRA needs a channel access scheme to specify when packets will join the $\mathrm{CR}$ process. The following definitions of two distinguishable parts of a queue are instrumental in describing the common channel access scheme we develop in this section. The primary queue of a node consists of packets that are to be transmitted in the next CRP, whereas other packets arriving at this particular node belong to the secondary queue. To avoid long CRP's, a shifting temporal window is used to limit the number of packets in the primary queue of each node. Just after a CRP, each node shifts its temporal window by a length of $\alpha_{0}$ to accept new packets to its primary queue from its secondary queue, provided that there are no packets waiting in the primary queue and no packet returns back to its queue from the previous CRP. If $p^{*}>0$, a packet, which cannot be resolved in a CRP, is assigned as the first element in the primary queue of the corresponding node and positions of waiting packets are shifted backwards by one.

To analyze the stability of CRA's, we use the notion of drift, which is defined as the expected length of a CRP less the expected change in $T(k)$. We first consider the case, when a CRP continues, until all packets in the primary queue of each node are successfully transmitted. Each node transmits its packets on first-come-first served basis. In the context of stability, this case is equivalent to the model of infinite node population. The only difference lies in the computation of the service time, which is a function of the number of nodes in the system. The negative drift condition for stability is expressed by the following inequality

$$
\frac{1}{\mu}<\alpha_{0}\left(1-p^{*}\right)
$$

In this system, a node will immediately try to transmit the 
second packet in its primary queue, whenever its first packet is successfully received. However, this second packet may cause a collision with any of the first packets of other nodes, which have not been resolved yet in the current CRP. This will destroy the standard structure of the CR tree and may cause successive collisions or idle slots, which will lead to the instability of the system. This system can be replaced with an inherently more stable one, where the transmissions during a single CRP are limited only to the first packets in the primary queue of each node. Since the latter approach we will follow throughout this section reduces the CRP length and prevents the proliferation of packets at each queue, it promises a relaxation of the stability condition and consequently increases the maximum achievable throughput.

Let $q_{i}$ be the number of packets at the primary queue of any node just before the $i^{\text {th }} \mathrm{CRP}$ with the probability mass function of $X_{i, k}=\operatorname{Prob}\left(q_{i}=k\right)$. The transient distributions of $q_{i}$ are given by the recursive equations (8) and (9) for $i \geq 0$ and $k \geq 0$, as described in Appendix A.

$$
\begin{aligned}
X_{i+1,0} & =\left(X_{i, 0}+X_{i, 1}\left(1-p_{i}^{*}\right)\right) e^{-a} \\
X_{i+1, k}= & \left(X_{i, 0}+X_{i, 1}\left(1-p_{i}^{*}\right)\right) \frac{a^{k} e^{-a}}{k !} \\
+ & X_{i, k+1}\left(1-p_{i}^{*}\right)+X_{i, k} p_{i}^{*}, k \geq 1
\end{aligned}
$$

where $a=\frac{\lambda \alpha_{0}}{m}$. The initial conditions for (8) and (9) are $X_{0, k}=\frac{a^{k} e^{-a}}{k !}$. The quantity $p_{i}^{*}$ denotes the probability that a packet cannot be successfully transmitted in the $i^{\text {th }}$ CRP and returns back to its queue. $p_{i}^{*}$ is a function of the number of nodes contending in the $i^{t h} \mathrm{CRP}$ and provides the only coupling between the values of the $X_{i, k}$ of different nodes. The quantity $p_{i}^{*}(n)$ is defined as the probability that a packet cannot be successfully transmitted in the $i^{\text {th }}$ CRP and, hence, returns back to its queue, provided that $n$ nodes are contending in the $i^{t h}$ CRP. Then, $p_{i}^{*}=\sum_{n=0}^{m} p_{i}^{*}(n) M_{n, i} . M_{n, i}$ is the probability mass function of a Bernouilli random variable that denotes the number of contending nodes $n$ in the $i^{t h}$ CRP and given by $M_{n, i}=\frac{m !}{n !(m-n) !}\left(X_{i, 0}\right)^{m-n}\left(1-X_{i, 0}\right)^{n}$. Note that $p_{i}^{*}(n)=p^{*}(n)$, for all values of $i$.

Let $X_{k}$ be the stationary probability that the number of packets in the queue of any node is $k$. Provided that the stationary distributions $X_{k}=\lim _{i \rightarrow \infty} X_{i, k}$ and $p_{i}^{*}=\lim _{i \rightarrow \infty} p_{i}^{*}$ exist for all $k$, the values of the $X_{k}$ 's are computed simply by replacing the quantities of $X_{i, k}$ and $p_{i}^{*}$ in (8) and (9) by the steady-state solutions $X_{k}$ and $p^{*}$ and by using the fact that $\sum_{k=0}^{\infty} X_{k}=1$. For the case $a<1$, the stationary distributions of $X_{k}$ 's take nonzero values expressed as in Eqs. (10) and (11).

$$
\begin{gathered}
X_{0}=\left(1+\frac{1}{1-p^{*}} \sum_{k=1}^{\infty} \sum_{j=k}^{\infty} \frac{a^{j}}{j !}\right)^{-1} \\
X_{k}=\frac{X_{0}}{1-p^{*}} \sum_{j=k}^{\infty} \frac{a^{j}}{j !} \quad, k \geq 1
\end{gathered}
$$

In Eqs. (10) and (11), $p^{*}=\lim _{i \rightarrow \infty} p_{i}^{*}=\sum_{n=0}^{m} p^{*}(n) M_{n}$. We have $p^{*}(0)=0, p^{*}(1)=0$ and the recursive equations for $p^{*}(n), n \geq 2$, are given in Eq. (12) as

$$
\begin{aligned}
p^{*}(n)= & B_{n, 0} p^{*}(n)+B_{n-1,1} B_{1,0} p^{*}(n-1) \\
& +\sum_{i=2}^{n-1} B_{n-1, i} B_{1,0} \\
& +\sum_{i=2}^{n} B_{n-1, i-1} B_{1,1} p^{*}(i)
\end{aligned}
$$

where $B_{n, i}$ denotes the probability that $i$ nodes (or equivalently $i$ packets) are assigned to the left branch at the first splitting in the CR tree, if $n$ nodes are contending for the channel. The quantity $B_{n, i}$ depends on the splitting criterion used by the underlying CRA. For instance, $B_{n, i}=\frac{n ! 0.5^{n}}{i !(n-i) !}$ in the case of binary fair splitting. The derivation of (12) is deferred to Appendix B. Note that our derivations were based on two assumptions:

1. The existence of steady-state distributions $X_{k}$ and $p^{*}$ (Numerical computations verify fast convergence of $X_{k, i}$ and $p_{i}^{*}$ to specific values of $X_{k}$ and $p^{*}$ depending on the value of a.)

2. The equality (with probability one) of long-term time averages of $p_{i}^{*}$ and $X_{i, k}$ with the corresponding ensemble averages

If the mean CRP length for conflicts of multiplicity $n$ is denoted as $L_{n}$, the expected service time $\frac{1}{\mu}$ is $\sum_{n=0}^{m} L_{n} M_{n}$. We have $L_{0}=1, L_{1}=1$, and the recursive equations for $L_{n}, n \geq 2$, are given in Eq. (13) for level skipping and in Eq. (14) for level skipping together with tree pruning, as

$$
\begin{gathered}
L_{n}=1-B_{n, 0}+2 \sum_{i=0}^{n} B_{n, i} L_{i} \\
L_{n}=1+B_{n, 1}\left(1+L_{n-1}\right)+B_{n, 0} L_{n}+\sum_{i=2}^{n} B_{n, i} L_{i}
\end{gathered}
$$

The closed form expressions for $L_{n}$ have been established in [13], [14] and [16]. In the asymptotic case, $\frac{L_{n}}{n}$ does not converge to a fixed value but fluctuates around $\frac{2}{\ln 2}$, as stated in [13], [14] and [17]. The mean of the CRP length illustrated in Figure 9 provides the necessary statistics about the CR process to express the stability criterion, as stated in Eq. (15).

$$
\lambda / m<\mu\left(1-X_{0}\right)\left(1-p^{*}\right)
$$

The quantity $\mu\left(1-X_{0}\right)\left(1-p^{*}\right)$ in Eq. (15) corresponds to the effective service rate, since a node successfully transmits a packet, if there exists at least one packet in its primary queue (with probability $1-X_{0}$ ) and the transmitted packet does not return back to its queue (with probability $1-p^{*}$ ). Figure 10 shows the relation between the maximum achievable throughput and the number of nodes $m$ for the best choice of $\alpha_{0}$ that maximizes the throughput for the given value of $m$.

\section{CONCLUSIONS}

In this paper, we addressed the CR problem in energylimited wireless networks and presented a residual-energybased CRA that increases throughput, extends system lifetime and first node death time, decreases the CRP length as well as the energy consumption per successfully transmitted packet for the case of a single cell. We identified the performance loss of the REBS algorithm in the particular case, when all nodes start operating with equal initial energy. To solve this problem partially, we proposed a hybrid approach that interchangeably uses the FCFS and REBS algorithms throughout the $\mathrm{CR}$ process, and showed via simulations that this method achieves desirable performance gains in the case of equal initial energy at the expense of increased complexity. We then 


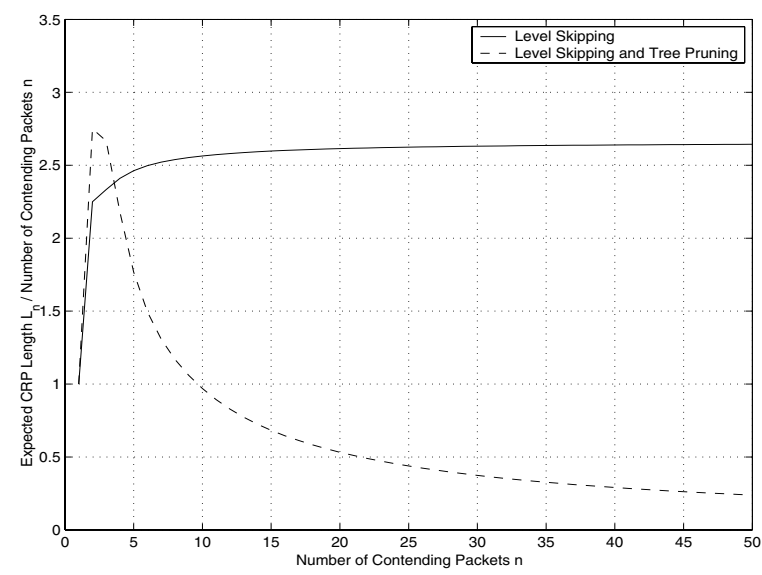

Fig. 9. $\frac{L_{n}}{n}$ as a function of the number of contending packets $n$ in the system for the case of infinite node energy and binary splitting

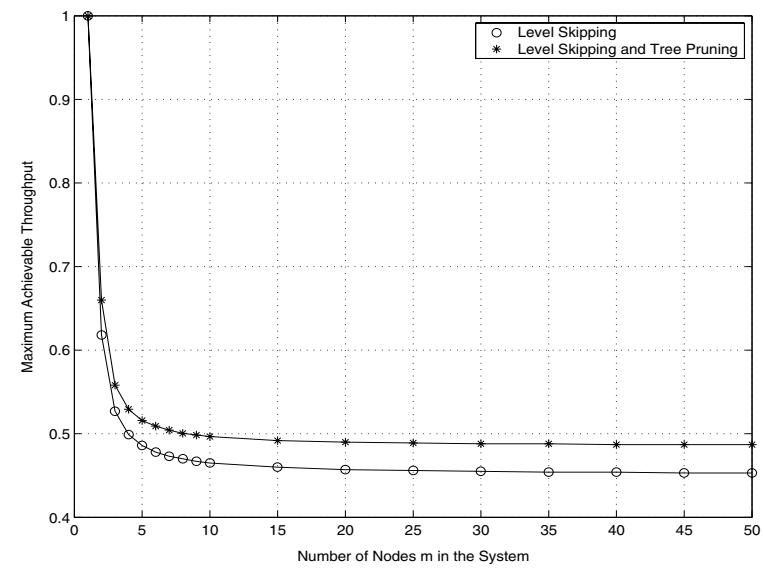

Fig. 10. Maximum achievable throughput as a function of the number of nodes $m$ for the case of infinite node energy and binary splitting

applied these ideas to energy-efficient operation for the case of unlimited energy supply, where instead of the residual energy we used the distance between transmitter and receiver as the criterion for splitting. Note that we used both the conventional collision channel as well as a physical model that takes into account SINR values. We also evaluated the performance under mobility (which is important for the case of physical model).

Furthermore, we extended the notion of residual-energybased conflict resolution to multi-hop ad-hoc networks and developed a simple method to reduce the interdependence between the simultaneous CR processes at different receivers, which might otherwise cause severe performance degradation. Further studies should concentrate on extending this CR treedecoupling method based on a simplified packet reception criterion to more sophisticated reception models as well as to networks with arbitrary number of nodes and arbitrary topologies. Finally, we looked at the CRA stability analysis of a single-cell system with a finite number of nodes by modeling it as a system of interacting queues. ${ }^{1}$

\section{REFERENCES}

[1] J. I. Capetanakis, "Tree Algorithms for Packet Broadcast Channels," IEEE Transactions on Information Theory, vol. 25, No. 5, pp. 505 -515, Sept. 1979

[2] D. Bertsekas and R. Gallager, Data Networks, 2nd ed. Prentice Hall, Englewood Cliffs, NJ (1992)

[3] B. S. Tsybakov and V. A. Mikhailov, "Free Synchronous Packet Access in a Broadcast Channel with Feedback," Probl. Pered. Inform., vol.14, No. 4, pp. 32-59, 1978

[4] M. L. Molle and G. C. Polyzos, "Conflict Resolution Algorithms and their Performance Analysis," Tec. Rep. Department of Computer Science and Engineering, University of California at San Diego, LaJolla, July 1993

[5] P. Gupta and P.R. Kumar, "The Capacity of Wireless Networks," IEEE Transactions on Information Theory, vol. 46, No.2, pp. 388-404, Mar. 2000

[6] A. Bruce McDonald and Taieb Znati, "A Mobility Based Framework for Adaptive Clustering in Wireless Ad-Hoc Networks," IEEE Journal on Selected Areas in Communications, vol. 17, No. 8, pp. 1466 -1487, Aug. 1999

[7] R. Knopp and P. A. Humblet, "Information Capacity and Power Control in Single-Cell Multiuser Communications," Int. Conf. on Communications, Seattle, Jun. 1995

[8] W. Luo and A. Ephremides, "Power Levels and Packet Lengths in Random Multiple Access," IEEE Transactions on Information Theory, vol. 48, No. 1 , pp. 46-58, Jan. 2002

[9] T. Saadawi and A. Ephremides, "Analysis, Stability, and Optimization of Slotted ALOHA with a Finite Number of Buffered Users," IEEE Transactions on Automatic Control, vol. AC-26, No. 3 , pp. 680-689, June 1981

[10] W. Szpankowski, "A Multiqueue Problem: Bounds and Approximations," in Performace of Computer-Communication Systems, Proc. IFIP Congr., 1984

[11] R. Rao and A. Ephremides, "On the Stability of Interacting Queues in a Multi-Access System," IEEE Transactions on Information Theory, vol. 34, No. 5, pp. 918-930, Sept. 1988

[12] W. Luo and A. Ephremides, "Stability of N Interacting Queues in Random-Access Systems," IEEE Transactions on Information Theory, vol. 45, No. 5, pp. 1579-1587, July. 1999

[13] P. Mathys and P. Flajolet, "Q-ary Collision Resolution Algorithms in Random-Access Systems with Free or Blocked Channel Access," IEEE Transactions on Information Theory, vol. 31, No. 2, pp. 217-243, Mar. 1985

[14] A. Janssen and M. Jong, "Analysis of Contention Tree Algorithms," IEEE Transactions on Information Theory, vol. 46, No. 6, pp. 21632172, Sept. 2000

[15] G. C. Polyzos and M. L. Molle, "A Queueing Theoretic Approach to the Delay Analysis for the FCFS 0.487 Conflict Resolution Algorithm," IEEE Transactions on Information Theory, vol. 39, No. 11, pp. 1887 -1906 , Nov. 1993

[16] J. L. Massey and P. Mathys, "The Collision Channel Without Feedback," IEEE Transactions on Information Theory, vol. 31, No. 2, pp. 192-204, Mar. 1985

[17] J. C. Huang and T. Berger, "Delay Analysis of Interval Searching Contention Resolution Algorithms," IEEE Transactions on Information Theory, vol. 31, No. 2, pp. 264-273, Mar. 1985

\section{APPENDIX}

\section{A. Derivation of the Transient Equations for $X_{i, k}$}

There is no packet in a queue of a particular node to be transmitted in the $(i+1)^{t h} \mathrm{CRP}$, if either the corresponding primary queue did not contain any packet before the previous CRP and no new packet arrived in the meantime (with probability $X_{i, 0} e^{-a}$ ) or the only packet transmitted from the primary queue was successfully received in the previous CRP

\footnotetext{
${ }^{1}$ The views and conclusions contained in this document are those of the authors and should not be interpreted as representing the official policies, either expressed or implied, of the Army Research Laboratory or the U.S.
} Government. 
and no new packet arrived in the meantime (with probability $\left.X_{i, 1}\left(1-p_{i}^{*}\right) e^{-a}\right)$. These two contributions to $X_{i, 0}$ can be combined in one expression as in the recursive equation (8). For $k \geq 1$, there are four distinct cases when the primary queue of a node (say node $j$ ) contains $k$ packets before the $(i+1)^{t h}$ CRP.

Case 1: The primary queue of node $j$ contained no packet to be transmitted for the next CRP and $k$ packets arrived during the $i^{\text {th }}$ CRP (with probability $X_{i, 0} \frac{a^{k} e^{-a}}{k !}$ ).

Case 2: In the $i^{t h}$ CRP, node $j$ transmitted the only packet in its primary queue that was successfully received and $k$ new packets arrived at its queue, as the $i^{\text {th }}$ CRP progresses (with probability $\left.X_{i, 1}\left(1-p_{i}^{*}\right) \frac{a^{k} e^{-a}}{k !}\right)$.

Case 3: The primary queue of node $j$ contained $k+1$ packets and one of them was successfully transmitted in the $i^{\text {th }}$ CRP (with probability $X_{i, k+1}\left(1-p_{i}^{*}\right)$ ).

Case 4: There were $k$ packets in the primary queue of node $j$ and the packet transmitted in the $i^{\text {th }}$ CRP returned back to its queue (with probability $X_{i, k} p_{i}^{*}$ )

The four cases can be combined in a unique expression for $X_{i+1, k}$ as in Eq. (9).

\section{B. Derivation of the Recursive Equation for $p^{*}(n)$}

Depending on the pattern of the first splitting after the collision of $n$ packets, there exist four distinct cases, when the packet of a particular node (say node $j$ ) is incorporated into the subsequent CRP, if we employ the tree pruning method, as described in section II-A.

Case 1: $n$ packets join the right branch (with probability $\left.B_{n, 0}\right)$ and the probability that the packet of node $j$ is incorporated into the next CRP is still $p^{*}(n)$.

Case 2: $n-1$ packets including the packet of node $j$ join the right branch, which occurs with probability $B_{n-1,1} B_{1,0}$, and the probability that the packet of node $j$ returns back to its queue in the given CRP is $p^{*}(n-1)$.

Case 3: Two or more packets are split into left branch and the packet of node $j$ joins the right branch. This occurs with probability $\sum_{i=2}^{n-1} B_{n-1, i} B_{1,0}$ and it is certain that the packet of node $j$ will return back to its queue.

Case 4: $i \geq 2$ packets (including the packet of node $j$ ) join the left branch with probability $\sum_{i=2}^{n} B_{n-1, i-1} B_{1,1}$. Then, the packet of node $j$ is incorporated into the next CRP with probability $p^{*}(i)$.

If we combine these four different cases in a unique expression, we end up with the recursive equation (12). A contending packet is successfully transmitted in a given CRP with probability one, if 0 or 1 node is simultaneously contending for the channel in this particular CRP. Therefore, the initial conditions for Eq. (12) are $p^{*}(0)=0$ and $p^{*}(1)=0$. 